\title{
Doppler ultrasound and photoplethysmographic assessment for identifying pregnancy-induced hypertension
}

\author{
XIURONG SUN ${ }^{1}$, FANGMING SU ${ }^{1}$, XUELIN CHEN $^{1}$, QIHUI PENG ${ }^{2}$, \\ XIAOMIN LUO $^{3,4^{*}}$ and XINGHAI HAO ${ }^{5}$
}

\begin{abstract}
Departments of ${ }^{1}$ Obstetrics and Gynecology and ${ }^{2}$ Ultrasonography, Shenzhen People's Hospital, Shenzhen, Guangdong 518020; ${ }^{3}$ Department of Healthcare, Beijing Genomics Institute, Shenzhen, Guangdong 518083; ${ }^{4}$ Tibet Branch, Beijing Genomics Institute, Lhasa, Tibet 850032;

${ }^{5}$ Department of Cardiac Surgery, Beijing Anzhen Hospital, Beijing 100029, P.R. China
\end{abstract}

Received March 24, 2019; Accepted October 28, 2019

DOI: $10.3892 /$ etm.2019.8405

\begin{abstract}
The current study investigated whether placentation and systemic inflammation are associated with pregnancy-induced hypertension $(\mathrm{PIH})$ or pre-eclampsia (PE), and evaluated some measurable indexes for assessment of maternal factors contributing to high-risk pregnancy. Photoplethysmographic reflection index (PPG RI), uterine artery (UtA) pulsatile index (PI) and reflection index (RI), as well as maternal serum placental growth factor (PlGF) and soluble endoglin (sEng) were measured in pregnant women with singleton pregnancy at the gestational age of 22 to 23 weeks. Study subjects were women with normal pregnancy $(N P, n=24)$, PIH $(n=14)$ and PE $(n=16)$. It was found that individuals in the PIH group exhibited higher UtA RI and UtA PI values, as well as PPG RI values compared with individuals in the NP group. Individuals in the PE group had the highest UtA RI, UtA PI and PPG RI values among these 3 groups. UtA and PPG results were significantly different in PIH and PE groups compared with the NP group. Significant differences were found in both PlGF and sEng levels between PIH and PE groups. A strong inverse across-subject correlation was found between PIGF and sEng levels. A weak inverse correlation was found between PIGF and UtA RI, and PIGF and UtA PI. A moderate inverse correlation was found between PlGF and PPG RI. A moderate positive correlation was found between either sEng and UtA RI or sEng and UtA PI. A very strong positive correlation was found between sEng and PPG RI. Taken together, the current results indicated that maternal effects related to cardiovascular adaptation to placentation and systemic inflammation exhibited significant differences between NP and PIH or PE groups.
\end{abstract}

Correspondence to: Dr Xiaomin Luo, Department of Healthcare, Beijing Genomics Institute, 11 Beishan Industrial Zone, Yantian Shenzhen, Guangdong 518083, P.R. China

E-mail: luo.xiaomin@139.com

Key words: pregnancy-induced hypertension, Doppler, photoplethysmography, pre-eclampsia, placentation
Therefore, assessment of UtA and PPG could be used for identifying high-risk pregnancy.

\section{Introduction}

During normal pregnancy (NP), the circulation of placenta is high-flow and low-resistance to meet the growth needs of the fetus. Inadequate or excessive cardiovascular system adaptation in pregnant women before 20 weeks of gestation is associated with pregnancy complications such as pregnancy-induced hypertension (PIH) and pre-eclampsia (PE) (1-3).

The main cause of death in pregnant women and fetuses is hypertensive syndrome in pregnancy, a common disease in obstetrics with an incidence of $3-10 \%$ (4). The disease is divided into gestational hypertension, mild pre-eclampsia, severe pre-eclampsia and eclampsia, with gestational hypertension being the least severe form and eclampsia being the most severe form. There have been a number of studies on hypertensive syndromes in pregnancy, but its etiology is not fully understood. So far, the potential pathological mechanisms of recognized PIH include: i) Abnormal invasion of maternal uterine blood vessels by the placental trophoblast; ii) intolerant maternal and fetal tissue immune molecules; and iii) genetic factors $(5,6)$. A combination of these factors results in decreased placental blood flow and oxygen supply and represses infiltrating cells after trophoblastic involvement $(7,8)$. Furthermore, these pathological mechanisms induce inflammatory reactions and local oxidative stress, leading to the release of inflammatory mediators and free radicals (9-11). These factors can activate a large number of neutrophils, directly or indirectly causing vascular endothelial damage, which eventually leads to the development of PIH. Thus, determining the major contributing factors of PIH, particularly placentation and systemic inflammatory states, would benefit in early screenings of PIH and PE.

In the current study, Doppler ultrasound was used to detect parameters of blood flow in the uterine arteries of pregnant women. The effects of cardiovascular function and structural adaptation on placentation were investigated. In addition, non-invasive photoplethysmography (PPG) was used to measure the effects of systemic inflammation (12-15). The 
results of Doppler ultrasonography and PPG measurements can be used for assessment of maternal factors that contribute to high-risk pregnancy.

\section{Subjects and methods}

Study subjects. The current study was performed at the Obstetrics Department of Shenzhen People's Hospital. Subjects were recruited from nulliparous women $(n=228)$ who attended their routine first and second trimester screening in the Ultrasound Laboratory. The inclusion criteria were: i) A singleton pregnancy; and ii) a gestational age of 22 to 23 weeks. All pregnancies were dated by crown-rump length measurement and last menstrual period. All subjects refrained from caffeine and drugs that could alter the cardiovascular system function on the day before the tests. The exclusion criteria were: i) Current or prior history of hypertension; ii) the use of regular medication; and iii) the development of complications during pregnancy. The diagnostic criteria of PIH and $\mathrm{PE}$ were in accordance with the Obstetrics and Gynecology of the People's Medical Publishing House (16). The study was approved by the Institutional Review Board of Shenzhen People's Hospital and all subjects had signed their written informed consent.

Measurements of the PPG reflection index (PPG RI). In the current study, PPG signals were measured using a HC2180-D research platform, an enhanced analytical system for data processing of physiological waveforms (Comperson Biotechnology Co., Ltd.), at a sampling rate of $500 \mathrm{~Hz}(8)$. PPG RI is derived from PPG amplitude changes of systolic and diastolic peak/inflection points in the PPG waveforms. PPG RI is defined as the ratio of the reflection peak amplitude to the pulse maximum amplitude (12). The equation for PPG RI was expressed as PPG RI=systolic peak amplitude/diastolic peak amplitude $\mathrm{x} 100$ (Fig. 1A), or in the absence of a diastolic peak, PPG RI=systolic peak amplitude/inflection point amplitude x100 (18-20) (Fig. 1B).

PPG signals were recorded from the right index finger of all subjects during resting state at a sitting position with the right hand being held at heart level. After the subjects rested for 5 min to ensure cardiovascular stability, PPG recordings were performed at a duration of $90 \mathrm{sec}$, with an averaging period covering at least $\geq 60$ pulse intervals. After a 5 -min rest, PPG signals were recorded again in a relaxed state, during which all the subjects were asked to calm down and breathe normally.

Measurements of uterine artery (UtA) pulsatile index (PI) and reflection index (RI). Blood flow velocity waveforms from both sides of the UtA in all subjects were obtained using a Philips iU22 Ultrasound system (Philips Medical Systems B.V.) with a 3.5- or 5-MHz probe. After PPG detection, Doppler ultrasound examinations of the transabdominal UtA were performed. UtA PI and RI were obtained immediately after nuchal translucency or anomaly fetal scan.

Upon the ultrasound examination, the UtA were identified in the oblique plane of pelvis, at the apparent crossover with the external iliac artery of its respective side. A previous study reported that examination of the UtA close to placental insertion revealed more diastolic flow and lower vascular resistance even under pathological conditions compared with healthy individuals (12). Hence, to standardize the sample site in the current study, the proximal part of the UtA was examined for UtA PI and UtA RI measurements. Pulsed-wave Doppler was applied to capture UtA Doppler flow velocity waveforms from which UtA PI and UtA RI were calculated automatically by the system. The following equations were used: UtA PI=(peak systolic velocity-end diastolic velocity)/mean velocity and UtA RI=(systolic maximal velocity-diastolic maximal velocity)/systolic maximal velocity (12).

Measurement of angiogenic proteins, including placental growth factor (PlGF) and soluble endoglin (sEng). The serum concentrations of PIGF and sEng of all participants were obtained from their medical records. PlGF (1:2; cat. no.DPG00) and sEng (1:5; cat. no. DNDG00) data recorded at the gestation week 22 (measured using R\&D Systems Inc. kits) were used in the analysis.

Statistical analysis. Statistical analyses were performed using SPSS 19.0 software (IBM Corp.). Results are presented as the mean \pm SD. Comparisons between groups were performed using a one-way ANOVA followed by Student-Newman-Keuls test. In addition, the effects of the diastolic and systolic state on the three subject groups were evaluated using the Pearson's product moment correlation coefficient for the vascular tone measured using PPG RI and the serum concentrations of PIGF and sEng. Strong correlation was defined as $r>\mid 0.81$, moderate correlation as $|0.8|>r>|0.3|$, and weak correlation as $r<|0.3|$.

The effect of cardiovascular adaptation on placentation was evaluated through the correlation between the Doppler ultrasound results of UtA PI and UtA RI, and the serum concentrations of PIGF and sEng.

\section{Results}

Clinicopathological characteristics. Among the participants studied, 14 developed PIH and 16 developed PE in the third trimester. These 30 patients were divided into two groups 6 weeks after delivery as follows: PIH participants $(n=14)$ and PE participants $(n=16)$. A total of 24 normotensive pregnant women were selected as a control group. The general characteristics of participants, including age, smoking status, ethnicity, height, body weight, pre-pregnancy body mass index (BMI), are shown in Table I.

$P I H$ and PE patients exhibits higher PPG RI values. Participants in PIH and PE groups exhibited significantly higher UtA RI, UtA PI and PPG RI values compared with the NP group (all $\mathrm{P}<0.05$; Table II). Analysis between PIH and $\mathrm{PE}$ groups revealed a statistically significant difference in the UtA PI and RI values $(\mathrm{P}<0.05)$, however, PPG RI values between the two groups did show any significant changes (Table II). The results indicated that UtA PI and RI and PPG data were distinct among NP, PIH and PE groups.

PIH and PE patients exhibits different PlGF and sEng levels. Additionally, the serum levels of PIGF and sEng between PIH and PE patients at week 22 showed a significant difference $(\mathrm{P}<0.05$; Table II). This result indicated that these two 

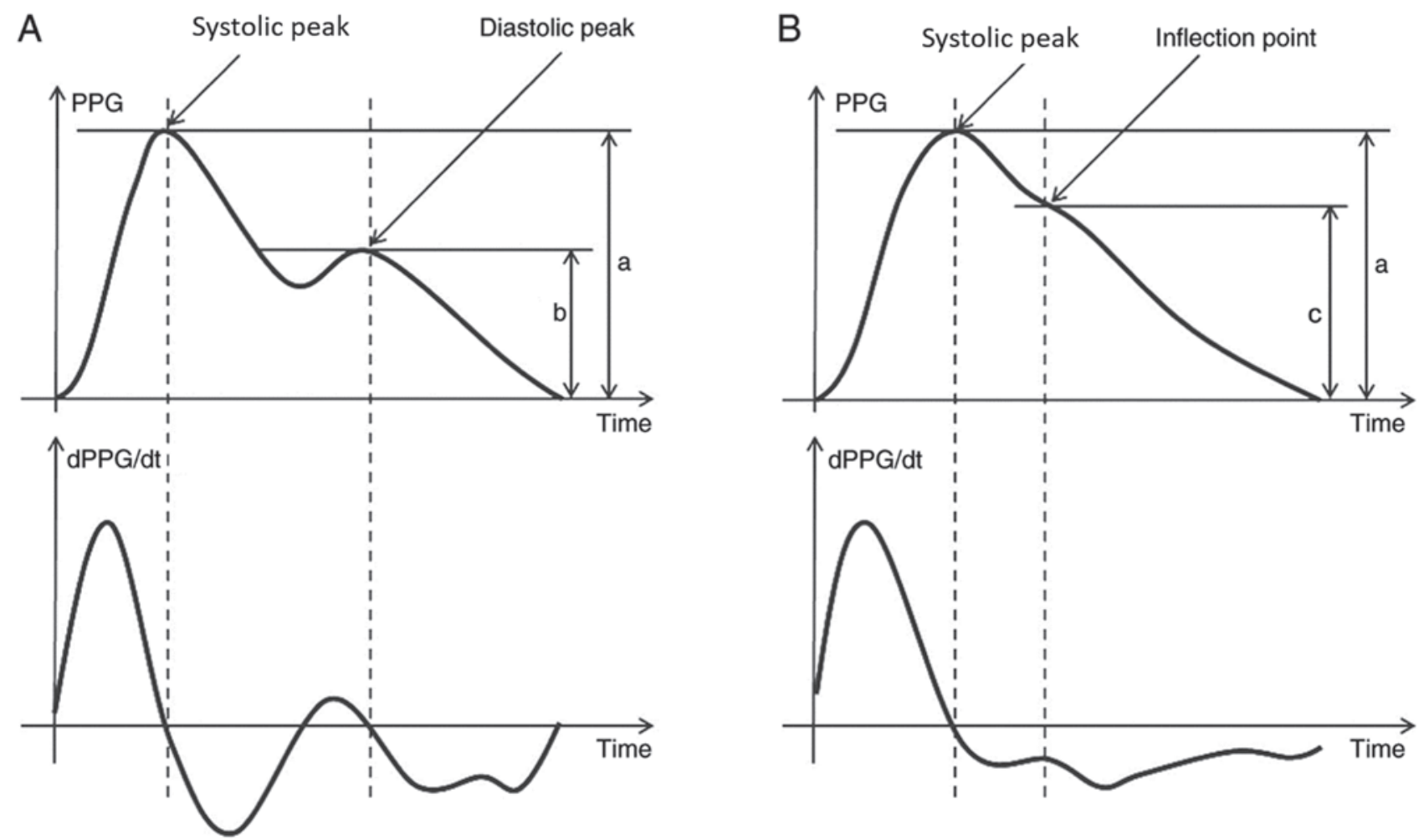

Figure 1. Normalized waveform and its dPPG/dt obtained using PPG exhibiting (A) a diastolic peak and (B) an inflection point. PPG, photoplethysmography; a, systolic peak amplitude; $b$, diastolic peak amplitude; c, inflection point amplitude; dPPG/dt, first derivative of normalized waveform.

Table I. Clinical characteristics of patients.

\begin{tabular}{lccc}
\hline Characteristic & NP $(\mathrm{n}=24)$ & $\mathrm{PIH}(\mathrm{n}=14)$ & $\mathrm{PE}(\mathrm{n}=16)$ \\
\hline Ethnicity & Chinese & Chinese & Chinese \\
Smoking status & no & no & no \\
Maternal age (years) & $26.46 \pm 4.29$ & $25.14 \pm 2.74$ & $27.06 \pm 3.64$ \\
Height $(\mathrm{cm})$ & $160.08 \pm 4.92$ & $160.07 \pm 3.25$ & $157.50 \pm 3.98$ \\
Weight $(\mathrm{kg})$ & $53.13 \pm 6.82$ & $51.71 \pm 7.55$ & $51.69 \pm 10.22$ \\
BMI $\left(\mathrm{kg} / \mathrm{m}^{2}\right)$ & $20.69 \pm 2.03$ & $20.15 \pm 2.66$ & $20.79 \pm 3.73$ \\
\hline
\end{tabular}

Values are presented as the mean $\pm \mathrm{SD}$. No significant difference was observed between groups.

Table II. PPG, Doppler ultrasonography, PIGF and sENG data obtained at week 22 of gestation.

\begin{tabular}{lrrr}
\hline Index & NP $(\mathrm{n}=24)$ & PIH $(\mathrm{n}=14)$ & \multicolumn{1}{c}{$\mathrm{PE}(\mathrm{n}=16)$} \\
\hline UtA RI & $0.49 \pm 0.07^{\mathrm{a}, \mathrm{b}}$ & $0.62 \pm 0.06^{\mathrm{b}, \mathrm{c}}$ & $0.69 \pm 0.09^{\mathrm{a} c \mathrm{c}}$ \\
UtA PI & $0.78 \pm 0.19^{\mathrm{a}, \mathrm{b}}$ & $1.19 \pm 0.26^{\mathrm{b}, \mathrm{c}}$ & $1.50 \pm 0.44^{\mathrm{a}, \mathrm{c}}$ \\
PPG RI & $0.44 \pm 0.09^{\mathrm{a}, \mathrm{b}}$ & $0.53 \pm 0.01^{\mathrm{c}}$ & $0.58 \pm 0.08^{\mathrm{c}}$ \\
PlGF & $320.81 \pm 68.38^{\mathrm{b}}$ & $312.21 \pm 90.01^{\mathrm{b}}$ & $254.25 \pm 53.32^{\mathrm{a}}$ \\
sEng & $5.78 \pm 1.16^{\mathrm{a}, \mathrm{b}}$ & $6.63 \pm 1.00^{\mathrm{b}, \mathrm{c}}$ & $7.48 \pm 1.05^{\mathrm{a}, \mathrm{c}}$ \\
\hline
\end{tabular}

Values are presented as the mean $\pm \mathrm{SD}$. ${ }^{\mathrm{a}} \mathrm{P}<0.05$ vs. $\mathrm{PIH}$; ${ }^{\mathrm{b}} \mathrm{P}<0.05$ vs. $\mathrm{PE}$; and ${ }^{\mathrm{C}} \mathrm{P}<0.05$ vs. NP. NP, normal pregnancy; $\mathrm{PIH}$, pregnancy-induced hypertension; PE, pre-eclampsia. UtA, uterine artery; RI, reflection index; PI, pulsatile index; PIGF, placental growth factor; sEng, soluble endoglin.

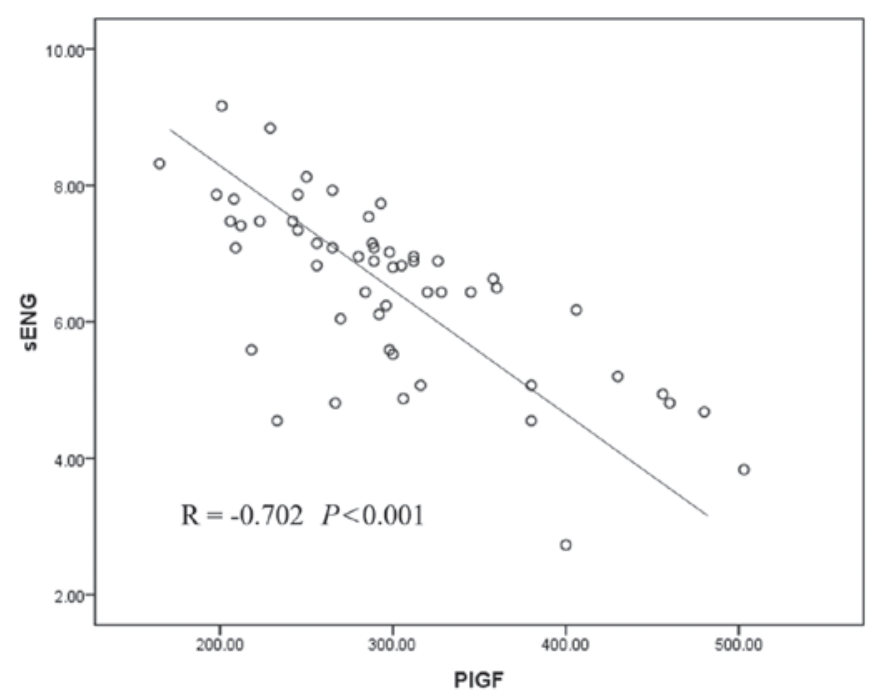

Figure 2. Correlation of PlGF and sEng in all subjects. $r=-0.702, \mathrm{P}<0.001$. PIGF, placental growth factor; sENG, soluble endoglin.

components might be involved in vascular regulation of blood circulation.

Correlation between PlGF, sEng, UtA PI, UtA RI, PPG RI values. An significant inverse across-subject correlation was found between PlGF and sEng serum levels ( $r=-0.702$, $\mathrm{P}<0.001$; Fig. 2). An inverse correlation was found between both PIGF and UtA PI values and PIGF and UtA RI values, while an inverse correlation was found between PlGF and PPG RI values $(r=-0.396, P=0.003 ; r=-0.378, P=0.005$; and 

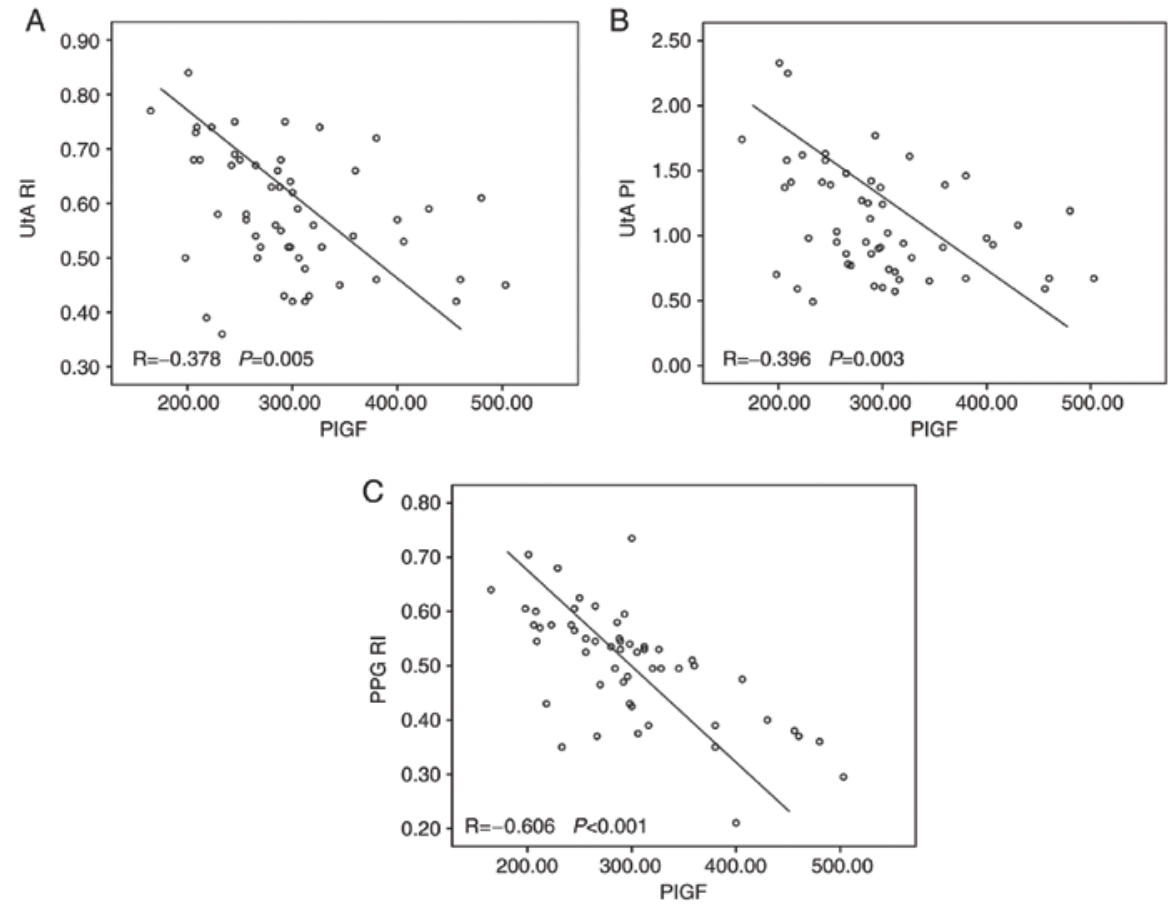

Figure 3. Correlation of PIGF and index values in all subjects using Pearson's product moment correlation coefficient. (A) Correlation of PlGF and UtA RI, $\mathrm{r}=-0.378, \mathrm{P}=0.005$. (B) Correlation of PlGF and UtA PI, $r=-0.396, \mathrm{P}=0.003$. (C) Correlation of PlGF and PPG RI, $r=0.606, \mathrm{P}<0.001$. RI, reflection index; PI, pulsatile index; PPG, photoplethysmography; PlGF, placental growth factor; sENG, soluble endoglin; UtA, uterine artery.
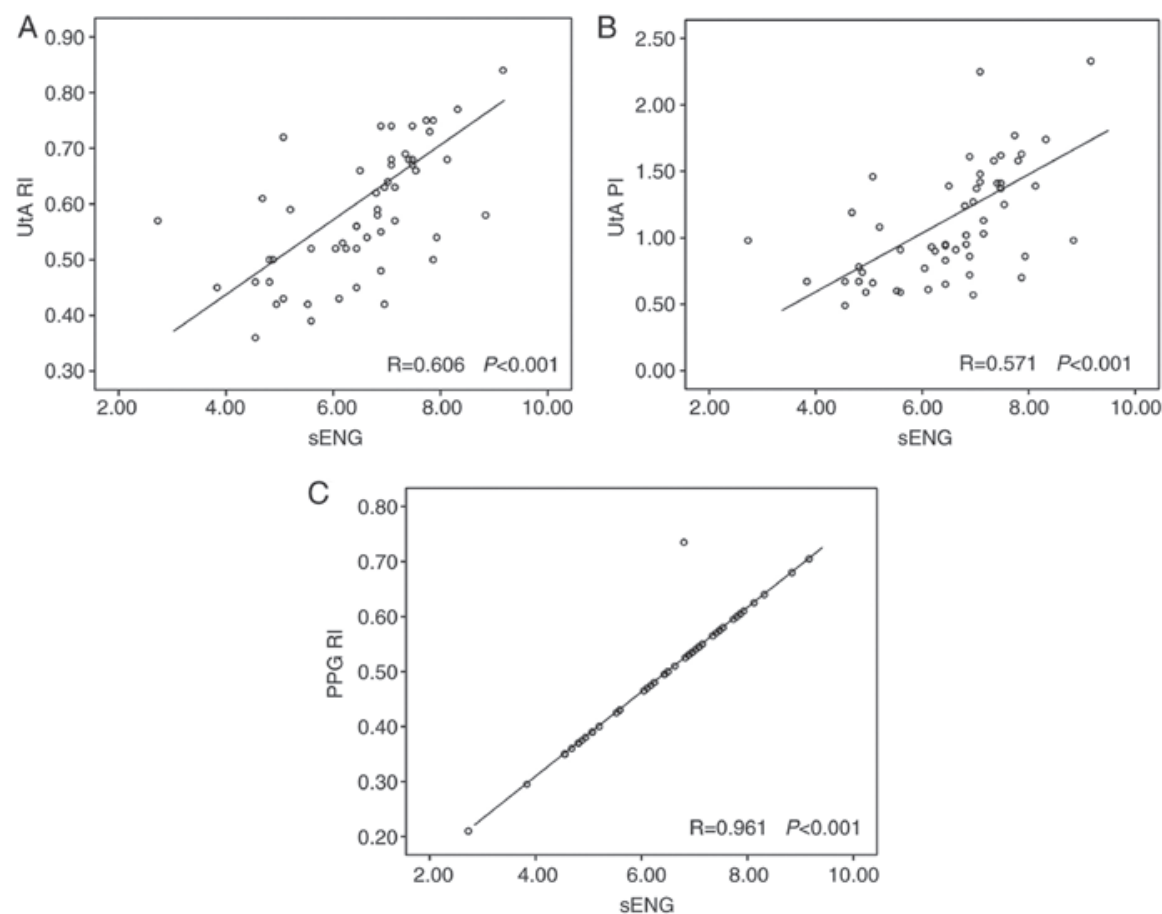

Figure 4. Correlation of sEng and index values in all subjects. (A) Correlation of sEng and UtA RI, $r=0.606, P<0.001$. (B) Correlation of sEng and UtA PI, $\mathrm{r}=0.571, \mathrm{P}<0.001$. (C) Correlation of sEng and PPG RI, r=0.961, P<0.001. RI, reflection index; PI, pulsatile index; PPG, photoplethysmography; PIGF, placental growth factor; sENG, soluble endoglin; UtA, uterine artery.

$\mathrm{r}=-0.606, \mathrm{P}<0.001$; respectively; Fig. 3A-C). A positive correlation was found between sEng and UtA RI values and sEng and UtA PI values $(r=0.606, P<0.001$; and $r=0.571$, $\mathrm{P}<0.001$; Fig. 4A and B). Additionally, a positive correlation was found between sEng and PPG RI values ( $\mathrm{r}=0.961$, $\mathrm{P}<0.001$; Fig. 4C).

\section{Discussion}

The occurrence of PE is commonly associated with large placentae or oxidatively-stressed placentae with multiple contributing factors (17). While poor placentation is considered a major predisposing factor for the occurrence of $\mathrm{PE}$, systemic 
inflammatory response is also considered to be a contributing factor of PE development, and may be caused by physiological shedding of apoptotic debris into the maternal circulation as part of normal renewal of the syncytiotrophoblast (18). In fact, PE might be the extreme end of a universal maternal response to pregnancy (9). If PE occurs, indicative measures to reflect the severity and the effects of systemic inflammation are needed throughout pregnancy to improve the assessment of the physiological condition of pregnancy, which is currently a limitation at prenatal clinics.

In the current study, UtA PI, UtA RI and PPG RI values exhibited inverse correlation with PIGF serum levels and a positive correlation with sEng serum levels. Both Doppler assessment of UtA PI and UtA RI, and PPG RI assessment were correlated with the maternal serum concentrations of PIGF and sEng. The correlation between PPG RI and PIGF and sEng serum concentrations suggested that the effect of vascular regulation may be used to estimate the circulatory state when the inflammatory response is activated. The relatively weaker correlation between UtA PI and UtA RI and the serum concentrations of PIGF and sEng, compared with other correlation comparisons included in the current study, suggested that the effects associated with poor placentation might be, to a certain degree, reflected and used as an estimation of defective placentation.

It remains to be confirmed whether the decreased PIGF levels observed in the current study directly represented increased inflammation. PIGF is an angiogenic molecule of the vascular endothelial growth factor family. In humans, serum levels of PIGF are reduced in women with PE $(19,20)$. Decreased PIGF levels are associated with increased levels of pro-inflammatory circulating interleukin (IL)-33 (21). Thus, pro-inflammatory molecules and cytokines may play a role in the pathogenesis of PE. IL-33 exerts its inflammatory action through its receptor interleukin-1 receptor-like 1 (22), which is expressed in the nuclei of endothelial cells of both large and small vessels, as well as in the placental endothelium and smooth muscle cells (23). In a previous study, an inverse correlation between IL-33 and PIGF was found both in PE and control groups, suggesting that cytokines were released when PlGF levels decreased (24). Numerous cytokines are released from the inter-villous space into the maternal circulation, causing systemic maternal disease (25).

As part of the adaptation process, normal pregnancy is characterized by systemic inflammation, oxidative stress, alterations in levels of angiogenic factors and vascular reactivity (18). Inflammation induced endothelial dysfunction and enhanced NO production and vasodilatation. The endothelium is a crucial regulator of the vascular tone. Impaired endothelial function is characterized by reduced vasodilation and increased vascular tone measured using PPG RI, represented as the amplitude of the reflected wave, which has been shown to correlate with the severity of proinflammatory and prothrombotic states (26). It is currently accepted that maternal endothelial dysfunction preceeds the development of PE (27). In the current study, increased sEng, which is associated with endothelial dysfunction, was found in PIH and PE groups compared with the NP group and was the highest in the PE group. An $r>0.9$ also suggested a very strong positive correlation between sEng and PPG RI.

The vascular tone measured using PPG RI increased in subjects with severe symptoms (28). Inflammation in vascular tissue is an important contributor to the pathophysiology of hypertension, the initiation and progression of atherosclerosis, as well as the development of cardiovascular diseases (29-31). During pregnancy, PPG RI provides a simplified indication of the inflammatory response through which the inflammation severity is expressed as the magnitude within the range of universal maternal intravascular inflammatory response to pregnancy (32). It has also been verified that the alteration in PPG RI is a useful assessment to reflect the status of endothelial function $(33,34)$.

In summary, using noninvasive measures to reflect circulatory status, PPG RI provided a scale covering a wide range of conditions, from normal to abnormal pregnancy. The UtA PI and RI determined using Doppler ultrasonography demonstrated the effects associated with poor placentation. These measures are useful in assessing high-risk pregnancy.

\section{Acknowledgements}

Not applicable.

Funding

No funding was received.

\section{Availability of data and materials}

The datasets used and/or analyzed during the current study are available from the corresponding author on reasonable request.

\section{Authors' contributions}

XL was responsible for research conception and design. FS, $\mathrm{XC}$ and $\mathrm{XH}$ are responsible for data acquisition. Data analysis and interpretation was performed by QP and XS. The article was written by XS and critically revised by XS and XH. All authors approved the final manuscript.

\section{Ethics approval and consent to participate}

The present study was approved by the Institutional Review Board of Shenzhen People's Hospital and all subjects had signed written informed consent.

\section{Patient consent for publication}

Not applicable.

\section{Competing interests}

The authors declare that they have no competing interests.

\section{References}

1. Kintiraki E, Papakatsika S, Kotronis G, Goulis DG and Kotsis V: Pregnancy-induced hypertension. Hormones (Athens) 14: 211-223, 2015.

2. Liu FM, Zhao M, Wang M, Yang HL and Li L: Effect of regular oral intake of aspirin during pregnancy on pregnancy outcome of high-risk pregnancy-induced hypertension syndrome patients. Eur Rev Med Pharmacol Sci 20: 5013-5016, 2016.

3. Draganovic D, Lucic N and Jojic D: Oxidative stress marker and pregnancy induced hypertension. Med Arch 70: 437-440, 2016. 
4. Damodaran D: Effect of progressive muscle relaxation technique in terms oi anxiety and physiological parameters of antenatal mothers with pregnancy-induced hypertension. Nurs J India 106 254-257, 2015.

5. Leffert LR, Clancy CR, Bateman BT, Bryant AS and Kuklina EV: Hypertensive disorders and pregnancy-related stroke: Frequency, trends, risk factors, and outcomes. Obstet Gynecol 125: 124-131, 2015.

6. Naderi S, Tsai SA and Khandelwal A: Hypertensive disorders of pregnancy. Curr Atheroscler Rep 19: 15, 2017.

7. Davenport MH, Ruchat SM, Poitras VJ, Jaramillo Garcia A, Gray CE, Barrowman N, Skow RJ, Meah VL, Riske L, Sobierajski F, et al: Prenatal exercise for the prevention of gestational diabetes mellitus and hypertensive disorders of pregnancy: A systematic review and meta-analysis. Br J Sports Med 52: $1367-1375,2018$

8. Sultana Z, Maiti K, Dedman L and Smith R: Is there a role for placental senescence in the genesis of obstetric complications and fetal growth restriction? Am J Obstet Gynecol 218: S762-S773, 2018.

9. Phipps E, Prasanna D, Brima W and Jim B: Preeclampsia: Updates in pathogenesis, definitions, and guidelines. Clin J Am Soc Nephrol 11: 1102-1113, 2016.

10. Jim B and Karumanchi SA: Preeclampsia: Pathogenesis, prevention, and long-term complications. Semin Nephrol 37: 386-397, 2017.

11. Guedes-Martins L: Superimposed preeclampsia. Adv Exp Med Biol 956: 409-417, 2017

12. Liu Z, Zhou Y, Yi R, He J, Yang Y, Luo L, Dai Y and Luo X Quantitative research into the deconditioning of hemodynamic to disorder of consciousness carried out using transcranial Doppler ultrasonography and photoplethysmography obtained via finger-transmissive absorption. Neurol Sci 37: 547-555, 2016.

13. Han N, Luo X and Su F: A quantitative investigation of hemodynamic adaptation to pregnancy using uterine artery Doppler ultrasonography and finger photoplethysmography. Hypertens Pregnancy 33: 498-507, 2014.

14. Di Santo P, Harnett DT, Simard T, Ramirez FD, Pourdjabbar A, Yousef A, Moreland R, Bernick J, Wells G, Dick A, et al: Photoplethysmography using a smartphone application for assessment of ulnar artery patency: A randomized clinical trial. CMAJ 190: E380-E388, 2018.

15. Ling P, Quan G, Siyuan Y, Bo G and Wei W: Can the descending aortic stroke volume be estimated by transesophageal descending aortic photoplethysmography? J Anesth 31: 337-344, 2017.

16. Veerbeek JH, Hermes W, Breimer AY, van Rijn BB, Koenen SV, Mol BW, Franx A, de Groot CJ and Koster MP: Cardiovascular disease risk factors after early-onset preeclampsia, late-onset preeclampsia, and pregnancy-induced hypertension. Hypertension 65: 600-606, 2015 .

17. Yang P, Dai A, Alexenko AP, Liu Y, Stephens AJ, Schulz LC, Schust DJ, Roberts RM and Ezashi T: Abnormal oxidative stress responses in fibroblasts from preeclampsia infants. PLoS One 9: e103110, 2014

18. Tannetta D, Masliukaite I, Vatish M, Redman C and Sargent I: Update of syncytiotrophoblast derived extracellular vesicles in normal pregnancy and preeclampsia. J Reprod Immunol 119 98-106, 2017.

19. Vieillefosse S, Guibourdenche J, Atallah A, Haddad B, Fournier T, Tsatsaris V and Lecarpentier E: Predictive and prognostic factors of preeclampsia: Interest of PlGF and sFLT-1. J Gynecol Obstet Biol Reprod (Paris) 45: 999-1008, 2016.
20. Erez O, Romero R, Maymon E, Chaemsaithong P, Done B, Pacora P, Panaitescu B, Chaiworapongsa T, Hassan SS and Tarca AL: The prediction of late-onset preeclampsia: Results from a longitudinal proteomics study. PLoS One 12: e0181468, 2017.

21. Enninga EA, Nevala WK, Creedon DJ, Markovic SN and Holtan SG: Fetal sex-based differences in maternal hormones, angiogenic factors, and immune mediators during pregnancy and the postpartum period. Am J Reprod Immunol 73: 251-262, 2015.

22. Chen H, Zhou X, Han TL, Baker PN, Qi H and Zhang H: Decreased IL-33 production contributes to trophoblast cell dysfunction in pregnancies with preeclampsia. Mediators Inflamm 2018: 9787239, 2018.

23. Romero R, Chaemsaithong P, Tarca AL, Korzeniewski SJ, Maymon E, Pacora P, Panaitescu B, Chaiyasit N, Dong Z, Erez O, et al: Maternal plasma-soluble ST2 concentrations are elevated prior to the development of early and late onset preeclampsia-a longitudinal study. J Matern Fetal Neonatal Med 31: 418-432, 2018.

24. Stampalija T, Chaiworapongsa T, Romero R, Chaemsaithong P, Korzeniewski SJ, Schwartz AG, Ferrazzi EM, Dong Z and Hassan SS: Maternal plasma concentrations of sST2 and angiogenic/anti-angiogenic factors in preeclampsia. J Matern Fetal Neonatal Med 26: 1359-1370, 2013.

25. Granne I, Southcombe JH, Snider JV, Tannetta DS, Child T, Redman CW and Sargent IL: ST2 and IL-33 in pregnancy and pre-eclampsia. PLoS One 6: e24463, 2011.

26. Mishra RC, Rahman MM, Davis MJ, Wulff H, Hill MA and Braun AP: Alpha ${ }_{1}$-adrenergic stimulation selectively enhances endothelium-mediated vasodilation in rat cremaster arteries. Physiol Rep 6: e13703, 2018.

27. Possomato-Vieira JS and Khalil RA: Mechanisms of endothelial dysfunction in hypertensive pregnancy and preeclampsia. Adv Pharmacol 77: 361-431, 2016.

28. Couceiro R, Carvalho P, Paiva RP, Henriques J, Quintal I, Antunes M, Muehlsteff J, Eickholt C, Brinkmeyer C, Kelm M and Meyer C: Assessment of cardiovascular function from multi-Gaussian fitting of a finger photoplethysmogram. Physiol Meas 36: 1801-1825, 2015.

29. Khaddaj Mallat R, Mathew John C, Kendrick DJ and Braun AP: The vascular endothelium: A regulator of arterial tone and interface for the immune system. Crit Rev Clin Lab Sci 54: 458-470, 2017.

30. Gray SP and Jandeleit-Dahm KA: The role of NADPH oxidase in vascular disease - hypertension, atherosclerosis \& stroke. Curr Pharm Des 21: 5933-5944, 2015.

31. MadonnaR and De CaterinaR: Aquaporin-1 and sodium-hydrogen exchangers as pharmacological targets in diabetic atherosclerosis. Curr Drug Targets 16: 361-365, 2015.

32. Redman CW, Sacks GP and Sargent IL: Preeclampsia: An excessive maternal inflammatory response to pregnancy. Am J Obstet Gynecol 180: 499-506, 1999.

33. Kudaravalli J: Improvement in endothelial dysfunction in patients with systemic lupus erythematosus with $\mathrm{N}$-acetylcysteine and atorvastatin. Indian J Pharmacol 43: 311-315, 2011.

34. Rosato E, Barbano B, Gigante A, Aversa A, Cianci R, Molinaro I, Quarta S, Pisarri S, Afeltra A and Salsano F: Erectile dysfunction, endothelium dysfunction, and microvascular damage in patients with systemic sclerosis. J Sex Med 10: 1380-1388, 2013. 